Meta

Journal des traducteurs

Translators' Journal

\title{
Débat du siècle : fidélité ou recréation
}

\section{Yuan Xiaoyi}

Volume 44, numéro 1, mars 1999

Théorie et pratique de la traduction en Chine

The Theory and Practice of Translation in China

URI : https://id.erudit.org/iderudit/004633ar

DOI : https://doi.org/10.7202/004633ar

Aller au sommaire du numéro

\section{Éditeur(s)}

Les Presses de l'Université de Montréal

ISSN

0026-0452 (imprimé)

1492-1421 (numérique)

Découvrir la revue

\section{Citer cet article}

Xiaoyi, Y. (1999). Débat du siècle : fidélité ou recréation. Meta, 44(1), 61-77. https://doi.org/10.7202/004633ar

\section{Résumé de l'article}

C'est au début de ce siècle que la Chine a commencé, de façon systématique, à présenteret à traduire des oeuvres littéraires. Et maintenant que le siècle tire à sa fin, la querelle surles notions de fidélité et de recréation, qui s'est engagée dès le début de la pratiquetraduisante, semble s'envenimer. Cette opposition conceptuelle fait de la fidélité uncertain critère mécanique de la traduction littéraire. Pourtant, la notion de fidélité n'existequ'en s'appuyant sur son verso à la recréation. Et il n'est pas exagéré de dire que c'estjustement avec la recréation que la notion de fidélité peut devenir une base théorique àpartir de laquelle nous pouvons réfléchir sur certains problèmes concernant surtout lanature de la traduction littéraire. Loin d'avoir la prétention de résoudre les paradoxesexistant dans la traduction littéraire, nous voudrions seulement leur donner une explicationraisonnable et acceptable. Laissons ces paradoxes continuer à être paradoxaux : s'ilsn'existaient pas dans l'histoire de la traduction, notre étonnement n'aurait aucun sens. 


\title{
Débat du siècle: fidélité ou recréation
}

\author{
yuan xiaoyi \\ Université de Nanjing, \\ Nanjing, Chine
}

\begin{abstract}
RÉSUMÉ
C'est au début de ce siècle que la Chine a commencé, de façon systématique, à présenter et à traduire des œuvres littéraires. Et maintenant que le siècle tire à sa fin, la querelle sur les notions de fidélité et de recréation, qui s'est engagée dès le début de la pratique traduisante, semble s'envenimer. Cette opposition conceptuelle fait de la fidélité un certain critère mécanique de la traduction littéraire. Pourtant, la notion de fidélité n'existe qu'en s'appuyant sur son verso - la recréation. Et il n'est pas exagéré de dire que c'est justement avec la recréation que la notion de fidélité peut devenir une base théorique à partir de laquelle nous pouvons réfléchir sur certains problèmes concernant surtout la nature de la traduction littéraire. Loin d'avoir la prétention de résoudre les paradoxes existant dans la traduction littéraire, nous voudrions seulement leur donner une explication raisonnable et acceptable. Laissons ces paradoxes continuer à être paradoxaux: s'ils n'existaient pas dans l'histoire de la traduction, notre tâtonnement n'aurait aucun sens.
\end{abstract}

\section{ABSTRACT}

In China, regular publication of literary translation began at the turn of the century. Today, at the end of the century, the age-old debate opposing fidelity and recreation is very much alive. Fidelity has become a somewhat mechanical criterion for judging literary translation. Yet the notion of fidelity cannot exist without that of recreation. It is reasonable to suggest that it is precisely through recreation that fidelity could provide a theoretical basis for discussing certain literary translation problems. The aim of this article is not to resolve the paradoxes existing in literary translation but to provide reasonable and plausible explanations, for without these paradoxes, our theory would be meaningless.

II n'est probablement pas, en matière de traduction littéraire, de querelle plus vieille que celle sur les notions de fidélité et de recréation et elle peut, à mesure que se développe la théorie de la traduction littéraire, se transformer en toutes sortes de dichotomies conceptuelles: forme / sens, style / contenu ou même, en ce qui concerne le traducteur (y compris aussi le théoricien de la traduction), école linguistique / école artistique ou - selon la proposition de Jean-René Ladmiral - sourciers / ciblistes.

II est vrai que la traduction est d'autant plus complexe qu'elle peut, comme la critique, «adopter devant une œuvre deux types d'attitudes tout à fait différentes», selon qu'elle considère le texte d'origine comme un «objet» ou un «sujet» (Genette 1966: 157). C'est-à-dire chercher un équivalent (mais qu'est-ce qu'un équivalent?) du texte dans un autre système de langue ou recréer la valeur poétique à partir du texte d'origine.

II n'est pas si simple de faire un choix. En fait, cette dichotomie n'est que le produit d'un certain dualisme conceptuel, elle est artificielle et non scientifique: 
quand on dit «fidèle», il paraît qu'on ne sait jamais à quoi on doit être fidèle; et quand on dit «recréation », notion proposée tout d'abord par l'école artistique, on ne sait non plus comment définir précisément cette notion et, par conséquent, comment éviter d'utiliser cette notion comme prétexte pour se dérober aux difficultés linguistiques de la traduction littéraire.

II en est de même pour la dichotomie sourciers / ciblistes, car si on est sourcier, on peut défendre la langue sourcière aussi bien que le texte «sourcier» en tant que «tout», comme le fait Henri Meschonnic; et si l'on est «cibliste», on peut défendre aussi la langue cible ou le texte cible (le texte cible est quand même difficile à défendre, parce qu'il est loin d'être un résultant avant l'opération traduisante du traducteur).

C'est surtout en accentuant cette dichotomie que la notion de fidélité est peu à peu devenue un critère mécanique pour presque tous les traducteurs, même pour les traducteurs qui défendent férocement la notion de recréation, parce qu'ils sentent toujours une certaine culpabilité de ne pas être fidèles quand ils «recréent» leur texte-traduction. La fidélité est même devenue une arme théorique pour reprocher aux traducteurs l'insuffisance de leurs connaissances linguistiques et cognitives.

Le paradoxe est que cette notion, si importante et si souvent citée en théorie de la traduction, n'est cependant presque jamais formulée d'une façon complète. Notons que la plupart des théoriciens sont tentés de la définir et de l'expliquer par une comparaison. Tout comme Georges Mounin le dit (1955: 25) : «Les comparaisons sont boiteuses», mais «elles frappent, entrent dans l'esprit». Et «que valent ces comparaisons?» Que vaut, par exemple, la maîtresse belle mais infidèle de M énage?

La Chine connaît aussi, depuis sa première vague de traduction religieuse, cette vieille querelle entre la fidélité et la recréation. Et, le paradoxe reste le même, la notion de fidélité existe toujours comme un critère de la traduction ou l'équivalent de la traduction littérale, tandis que celle de recréation existe toujours comme moyen de se débarrasser des difficultés dues à la différence entre les deux langues et les deux cultures. Ici, nous voudrions, une fois de plus, citer le débat très significatif concernant les traductions chinoises du Rouge et le Noir, qui a eu lieu en 1995, année où cette querelle s'est exacerbée une fois de plus. Hao Yun, un traducteur qui insiste beaucoup sur l'importance de la fidélité, affirme: «Si l'original est une al gue, j'essaye del'offrir avec son goût original aux lecteurs chinois, sans la changer en nouilles, bien que l'algue soit peut-être plus difficile à digérer que les nouilles pour les lecteurs»; alors que Xu Yuanchong, un autre traducteur du même roman et un représentant de l'école artistique, va si loin qu'il croit que la traduction est une sorte de concurrence avec la langue de départ et la culture de départ ${ }^{1}$.

Le présent travail, tout en se demandant à quelle vérité artistique la traduction doit obéir, tâchera de réfléchir, sous l'angle de ces deux notions qui ne sont pas du tout antithétiques (c'est vrai, elles sont plutôt, comme la comparaison de Saussure, le recto et le verso d'une feuille de papier), sur quelques problèmes fondamentaux de la traduction littéraire. Et nous voulons signaler ici que nous discutons seulement du cas de la traduction littéraire. II existe certainement d'autres genres de traductions qui n'entrent pas dans le cadre de notre discussion. 


\section{LA FIDÉLITÉ : UN ANGLE THÉORIQUE DE LA TRADUCTION LITTÉRAIRE}

En effet, il n'est pas exagéré de dire que c'est justement la notion de fidélité qui soulève le problème de l'intraduisibilité, c'est-à-dire que la traduction n'est pas possible seulement parce qu'elle ne peut pas être fidèle, à tous ses niveaux et dans tous ses aspects.

Georges Mounin a classé les arguments de l'intraduisibilité en deux grandes catégories, l'une à cause des vertus propres à chaque langue, c'est-à-dire celles «des mots propres, des sentences et des énergies, de la magnificence des mots, de la gravité des sentences, etc. »; et l'autre à cause des raisons propres à chaque auteur en particulier, c'est-à-dire l'utilisation, spécifique à chaque auteur, de «tout ce qui touche au style» (1955: 33). C'est à partir de cette classification qu'il justifie la possibilité de la traduction en cherchant des arguments - dans la linguistique, bien sûr. Cependant, le problème est loin d'être résolu, car si l'on peut être fidèle à la sémantique, à la morphologie, à la phonétique, même à la stylistique séparément, on n'arrive jamais à réaliser toutes ces fidélités en même temps.

Bien sûr, en tant que pratique très ancienne du monde, son existence peut justifier elle-même la traduisibilité dans la pratique. Personne ne peut nier la nécessité et la fonction de la traduction, littéraire ou non. M ais la nécessité ne peut pas signifier la possi bilité au niveau philosophique: c'est là notre problème, d'où le fossé profond entre la pratique traduisante et la théorie de la traduction. Quand les traducteurs se demandent: «Imagine-t-on une autre activité humaine comparable par son importance, son étendue, sa pérennité, voir nier son existence en droit, au mépris des réalités quotidiennement constatables en fait?» (Ladmiral 1994: 85), et quand les historiens de la traduction disent que la langue cible «ne recevra toute sa perfection qu'en allant chez ses voisins pour commercer et pour reconnaître ses vraies richesses; en fouillant dans l'Antiquité à qui elle doit son premier levain et en cherchant les limites qui la séparent des autres langues» et affirment que «la traduction seule lui rendra de tels services» (Mounin 1955: 31-33), on risque aussi de tomber dans le «cercle absolu » (au sens d'Antoine Berman) qu'est la traduction.

Donc, il est temps de définir cette notion, non comme un critère absolu de l'éthique, mais comme un vrai angle théorique à travers lequel on peut examiner les problèmes fondamentaux de la traduction.

En effet, ce que la fidélité demande, c'est l'intelligence et le courage. C'est-à-dire que, d'une part, on doit harmoniser les fidélités de tous les niveaux (de pensée, sémantique et esthétique, comme le propose Xu Jun [1992: 1-15]) et que, d'autre part, on ne doit pas se dérober aux obstacles dus à la différence entre les deux langues et les deux cultures. Meschonnic a certainement raison de considérer le texte d'origine comme un ensemble esthétique insécable, et notre notion de fidélité ne peut négliger, non plus, ce point important. Nous devons faire remarquer au moins les trois points suivants:

1.1. Être fidèle, c'est tout d'abord une «habitude» philosophique de l'humanité. Bien que nous ayons déjà commencé à douter de la fameuse «imitation » d'A ristote, nous ne pouvons nier, une fois pour toutes, cette affirmation qui a influencé, et qui influence encore, notre histoire humaine. L'original, y compris notamment le texte 
d'origine de la traduction, est le seul «objet» que l'on peut toucher, sentir, analyser et considérer comme un tout. Le texte d'origine, une fois fini, fournit au traducteur au moins un point de départ temporairement statique. I miter ne veut pas dire copier, et ainsi l'opération traduisante n'est jamais un processus aussi simple que celui de «ranger des habits dans des bagages différents». Tout comme l'être humain n'est pas la simple somme mécanique des parties du corps, elle n'est pas non plus la somme mécanique des parties transposées de l'œuvre originale, la somme de «forme + contenu », ou bien de «style + sens». Ce point de départ n'est peut-être pas l'achèvement de la traduction, il a besoin, lui aussi, d'un esprit de création pour que sa vie puisse renaître dans un autre système de langue et dans une autre culture. La notion de fidélité nous rappelle l'importance de la tradition qui est la source de la création, et n'importe quelle sorte de création commence toujours par une certaine imitation. Si la traduction abandonne son point de départ, elle perdra toutes ses raisons d'être.

1.2. Et, ensuite, si la dichotomie fidélité / recréation se trouve au niveau conceptuel, nous devons quand même la différencier de celle qui se produit entre la traduction littérale et la traduction libre. Ici, nous trouvons que Amparo Albir a raison de dire que le «mot-à-mot» (ou traduction littérale) et la traduction libre sont, tous deux, des moyens de traduction coexistants, et il y en a certainement d'autres. M ême dans l'histoire de la traduction littéraire, certains traducteurs et théoriciens de la traduction ne voulaient plus assimiler la fidélité à la conception de «mot-à-mot»: quand Leconte de Lisle entendait par fidélité le fait de «conserver dans la traduction les façons de penser, sentir, parler, agir, vivre»; ou quand Xu Yuanchong, tout en défendant sa théorie de «concurrence», croit aussi qu'il est fidèle à l'œuvre originale de Stendhal. Le problème vient justement de là: si l'on veut insister sur la notion de fidélité, comment la différencier de la conception du «mot-à-mot» que presque aucun traducteur n'adopte plus aujourd'hui ? En réalité, les différences entre les deux systèmes de langues et les deux cultures ne permettent pas l'existence de la traduction tout à fait littérale ou de la traduction tout à fait libre. Dans une traduction qui n'a pas un grand succès, on trouve, le plus souvent, un mélange de ces deux moyens extrêmes. Le débat lui-même les rend dans l'absolu. Si la fidélité est seulement au niveau lexical, comme l'affirment certains tenants du «mot-à-mot», quand on rencontre un mot intraduisible, alors toute la traduction sera impossible. Pourtant, c'est justement au niveau lexical que la fidélité ne peut pas se réaliser. Ferdinand de Saussure (1968) a démontré que «la partie conceptuelle de la valeur (d'un terme) est constituée uniquement par des rapports et des différences avec les autres termes de la langue», c'est-à-dire que la langue représente une certaine relation, et que c'est la tension de cette relation qui fait le langage. Donc, dans une traduction, si l'on veut être fidèle à la langue (la langue de départ et la langue d'arrivée), on peut seulement être fidèle à cette tension, car la relation qui unit le signifiant au signifié est arbitraire. Et c'est seulement entre les deux tensions que le problème de l'équivalence existe.

1.3. Enfin, ce qui nous semble le plus important, c'est sans doute de répondre à cette vieille question: fidèle à quoi ? Revenons aux deux catégories que Georges Mounin a définies pour nous. Nous serons, une fois de plus, en face d'un choix difficile: aux vertus particulières à la langue ou aux vertus particulière au style de l'auteur? Nous voyons que les grands écrivains ont souvent tendance à transgresser 
les règles langagières. Ainsi Joachim du Bellay penset-il que «la traduction serait impossible à cause, non plus de propriétés des langues elles-mêmes, mais à cause de la façon très particulière dont les écrivains, surtout les poètes, se servent des langues » (Mounin 1955). Ici, I'opposition dualiste entre la fidélité et la recréation se transforme tout de suite en une autre: Serons-nous fidèles à la langue commune ou aux moyens d'expressions spécifiques des écrivains (souvent plus grands que nous, les traducteurs) ? Pourrons-nous être fidèles, en même temps, à la langue (en plus, à la langue de départ ou à la langue d'arrivée) et au style?

En effet, aujourd'hui, les théoriciens français essayent toujours de répondre à cette question et ils adoptent différentes approches: linguistiques, textuelles ou philosophiques, par exemple. Mais la plupart des théoriciens se contentent encore d'une fidélité à un seul niveau ou d'un seul aspect. Seul Albir nous donne trois paramètres de la fidélité au sens: le «vouloir dire» de l'auteur, la langue d'arrivée et le destinataire de la traduction, et elle écrit (1990: 114) :

Ce triple rapport de fidélité - au vouloir dire de l'auteur, à la langue d'arrivée et au destinataire de la traduction - est indissociable. Si l'on ne reste fidèle qu'à un seul de ces paramètres et qu'on trahit les autres, on ne sera pas fidèle au sens. Une traduction qui n'est pas claire pour son destinataire ou qui présente des erreurs de langue n'est pas une traduction fidèle au sens.

Ses efforts ne sont certainement pas perdus. C'est presque la première fois qu'un théoricien de la traduction propose de considérer la notion de fidélité comme un angle de théorisation à partir duquel on peut entrer dans les problèmes fondamentaux de la traduction. Cependant, le problème est né de son invariant: le sens, car le sens est aussi une conception qui est assez difficile à définir. Et la théorie interprétative de Seleskovitch et Lederer, que l'auteur a choisie comme base théorique, a aussi tendance à simplifier la traduction littéraire et à négliger les contradictions intrinsè ques de la littérature elle-même. Tout en essayant d'affirmer ainsi ses points de vue sur les divergences des deux écoles théoriques de la traduction, l'auteur accentue, luimême, la dichotomie sens / forme.

En Chine, la systématisation de la théorie de la traduction n'a commencé que depuis peu, mais la querelle entre la fidélité et la recréation existe depuis le commencement de la pratique traduisante. Le principe de ne point embellir l'original proposé par Zhiqian, les cinq cas de trahison de l'original, évoqués par Dao An, sont les exemples qui insistent sur la notion de fidélité. Et Yan Fu, qui a formulé les trois principes «la fidélité, l'expressivité et l'élégance», tout en mettant la fidélité au premier plan, a déjà essayé de réfléchir aux autres problèmes fondamentaux que la notion de fidélité ne pouvait pas englober. M ais la juxtaposition de ces trois principes ne peut pas, elle non plus, résoudre ce problème. Et Fu Lei, un grand traducteur contemporain de la langue française, se demande aussi à quelle vérité la traduction littéraire doit obéir : à l'esprit ou au corps? M ême aujourd'hui, dans le débat sur la traduction chinoise du Rouge et le N oir, la plupart des traducteurs et des théoriciens de la traduction se chicanent encore sur ce vieux problème de fidélité. Quand Xu Yuanchong traduit la dernière phrase du Rouge et le Noir : « $\mathrm{M}$ me de Rênal fut fidèle à sa promesse. Elle ne chercha en aucune manière à attenter à sa vie; mais trois jours après Julien, elle mourut en embrassant ses enfants» en 《德雷纳夫人忠于她的诺言, 没有自寻短见，但在于连死后三天，她也吻着 孩子，魂归离恨天了》, les autres 
traducteurs, les théoriciens et même les lecteurs sont choqués par cette phrase que le traducteur emprunte au titre du récit XCVIII du Rêve dans le pavillon rouge: "L'âme de la pauvre sœurette aux perles pourpres / revient se fondre au ciel des regrets abolis. » Le reproche vient aussi de son infidélité, seulement parce qu'il utilise une expression typiquement chinoise. «Si nous traduisons de façon directe cette traduction chinoise en français», certains théoriciens critiquent: "Ça doit être "I'âme de $M$ me de Rênal qui revient se fondre au ciel des regrets abolis", mais pas "mourut" du texte original »; ils font ainsi de la traduction littéraire un processus chimique de réduction. Et le réducteur, c'est justement cette notion mécanique de fidélité.

Préciser un invariant dans le processus de la traduction, c'est presque le rêve de tous les théoriciens et de tous les traducteurs. Pourtant la notion de fidélité, ce à quoi on doit être fidèle, comme nous venons de le montrer, peut difficilement rester un invariant. Ce paradoxe (c'est peut-être le paradoxe éternel de l'humanité) sera-t-il toujours impossible à «dialectiser »?

\section{LA RECRÉATION : L'AUTRE FACE DE LA FIDÉLITÉ}

C'est la nature de la traduction qui décide de l'existence de la fidélité, qu'on le veuille ou non. Mais, comme nous le voyons, il existe des problèmes sans réponse dans la notion de fidélité. Pour les éclaircir, il est nécessaire d'introduire ici la notion de recréation, non comme le contraire de la fidélité et le moyen obligatoirement adopté quand on ne peut pas être fidèle, mais comme un autre angle complémentaire.

Mais qu'est-ce que la recréation? Ce n'est pas une question à laquelle nous pouvons répondre facilement. Si nous voulons définir cette notion et la distinguer de la notion de création que l'école artistique nous propose, il faudrait tout d'abord faire ressortir ce qui s'oppose à la notion de recréation dans la traduction littéraire.

Ce qui s'oppose à la notion de recréation, ce n'est pas la réalité objective de la traduction littéraire, ce n'est pas non plus son fondement linguistique, c'est, en premier lieu, la notion de reproduction que certains confondent avec celle de fidélité.

Longtemps, la traduction est restée une pratique sociale non théorisée et nous ne pouvons nous empêcher de nous demander pourquoi. Cependant, si nous comprenons l'attitude naïve que l'on garde envers la traduction - jusqu'à aujourd'hui, certains croient encore que nous pouvons reproduire des textes originaux pourvu que nous soyons bilingues - , cela ne nous semble pas si paradoxal. Ce que la reproduction demande, ce n'est évidemment pas une certaine théorie, c'est plutôt, comme tous les travaux artisanaux, une sorte de technique.

Comme nous l'avons montré dans la première partie, la traduction est bien plus qu'une somme. Et ce «plus», c'est ce que nous appelons «l'esprit de création ». C'est justement cet esprit de création qui distingue le traducteur du simple écrivant et le fait devenir un véritable écrivain (selon la définition de Roland Barthes). L'équivalent d'une œuvre originale ne nous attend jamais. C'est au moins à l'aide du «bricolage intellectuel » - si nous acceptons le concept de Lévi-Strauss - du traducteur que cet équivalent peut être recréé dans un autre système de langue.

Bien sûr, le répertoire de «l'univers instrumental» du «bricoleur», dit LéviStrauss, si étendu soit-il, «restelimité». Donc, l'ingénieur «interrogel'univers, tandis que le bricoleur s'adresse à une collection de résidus d'ouvrages humains, c'est-à-dire à un sous-ensemble de la culture» (cité par M eschonnic 1982: 341). C'est peut-être 
pourquoi nous mettons un «re» devant le mot «création ». Mais, au fond, en quoi consiste ce «re»? Peut-être pouvons-nous dire que ce qui s'oppose à la notion de recréation, c'est aussi la notion de ré-écriture. En effet, traduire un texte n'est généralement pas ré-écrire un tout autre texte - bien que le sujet, par exemple, reste le même - dans la langue d'arrivée.

La nature de la traduction décide aussi de l'existence de la notion de recréation. C'est la raison pour laquelle nous préférerions ici le mot «recréativité» qui nous rappelle que la traduction est avant tout un processus historique.

Paul de Man (1986), représentant de l'école de Yale (Yale School), pense que la «littérarité» d'un texte littéraire consiste à «mettre la fonction rhétorique au-dessus des fonctions grammaticale et logique». Il existe une certaine «correspondance naturelle» entre la grammaire et la logique, mais «une relation incertaine» entre la grammaire et la rhétorique. Cette tension potentielle «se coagulera au cours de la lecture».

Roland Barthes, lui aussi, dans S/Z (qui marque son tournant vers le déstructuralisme), analyse minutieusement la différence entre «les textes scriptibles» et «les textes lisibles» (1970: 9-10):

II faut donc choisir : ou bien placer tous les textes dans un va-et-vient démonstratif [...] les forcer à rejoindre inductivement la Copie dont on les fera ensuite dériver; ou bien remettre chaque texte, non dans son individualité, mais dans son jeu, le faire recueillir, avant même d'en parler, par le paradigme infini de la différence, le soumettre d'emblée à une typologie fondatrice, à une évaluation. [...] Notre évaluation ne peut être liée qu'à une pratique et cette pratique est celle de l'écriture.

Comme les lecteurs se trouvent dans l'évolution infinie de l'histoire, la structure et le sens des textes constituent le changement et l'ouverture de cette historicité. La relation entre signifiant et signifié n'est pas définie, nous sommes actifs devant ces textes: «l'enjeu du travail littéraire (de la littérature comme travail), c'est de faire du lecteur, non plus un consommateur, mais un producteur du texte» (Barthes 1970: 10).

Tout cela nous fait réfléchir de nouveau, au moins sur trois problèmes.

1. Qu'est-ce que la lecture, qui constitue la première étape de la traduction? La langue elle-même - et n'importe quelle langue - est incertaine d'après les points de vue du déstructuralisme. La littérature ne peut alors jamais être réduite à une information transparente, et tout langage de textes littéraires se fonde toujours sur les autres langages et les autres textes. Ainsi, toute lecture est une sorte de «lecture fausse», une déformation du texte original qui est interminable. La prépondérance absolue de l'auteur est donc menacée, car ce n'est pas lui qui décide de la vie de son œuvre, mais tout au contraire, c'est le lecteur, y compris notamment notre traducteur, qui en décide.

2. Qu'est-ce que l'écriture, qui constitue la deuxième étape de la traduction? Selon Barthes, nous pouvons choisir entre «les textes scriptibles» et «les textes lisibles», c'est-à-dire entre la «création » et la «copie». Mais le problème est: Pouvonsnous vraiment copier tout le texte original quand nous traduisons? Peut-être est-ce pourquoi les théoriciens comme Nida (cité par Meschonnic 1982) pensent qu'«en transférant le message d'une langue à une autre, c'est le contenu qui doit être conservé, à tout prix: la forme, excepté dans des cas spéciaux, comme la poésie, est largement secondaire». Si nous choisissons la «copie», nous ne pouvons que copier ce que nous pouvons copier tout en sacrifiant ce que nous ne pouvons pas copier (soi-disant les éléments secondaires). 
3. Quelle place devons-nous donner à la traduction qui se situe entre la lecture et la « relecture», entre la compréhension et la «re-compréhension »? Comment comprendre cette historicité que presque toute théorie de la traduction a négligée? La complexité de la traduction est due non seulement à la différence spatiale, mais aussi à la différence temporelle. Si nous acceptons ce que Barthes (1970: 9-10) dit sur l'interprétation du texte, nous pouvons aussi dire que la traduction, "c'est nous en train d'écrire, avant que le jeu infini du monde (le monde comme jeu) ne soit traversé, coupé, arrêté, plastifié par quelque système singulier qui en rebatte sur la pluralité des entrées, l'ouverture des réseaux, l'infini des langages», ce sont des «productions» (et non des «produits») (Barthes 1970).

Tous ces problèmes fondamentaux posés par la notion de recréation nous en dévoilent les points faibles inhérents à la notion de fidélité: ce qui manque à la fidélité, c'est l'historicité, car la fidélité implique toujours ce à quoi on doit être fidèle. Elle néglige souvent ce qui est derrière la traduction et considère ainsi la traduction comme une fin. Mais la recréation nous rappelle la fonction, le but et la tendance. Tout comme le préjugé que l'herméneutique moderne justifie, la traduction, avec la participation du traducteur de son propre chef, est certainement juste.

La traduction n'est qu'une des stations de la circulation de la compréhension. Tout comme le texte original, elle est loin d'être un résultat, une fin. Cela explique bien l'inévitabilité de la retraduction. La vie d'un texte ne s'arrête jamais à l'œuvre de I'auteur, bien que certains écrivains comme Milan Kundera se considèrent comme des personnes qui disent le dernier mot. Mais elle participe aussi de ses lecteurs, de ses traducteurs qui font sortir le texte original de ses limites temporelles et spatiales. Le lien entre le signifiant et le signifié n'est pas fixé, il est ouvert à tous les lecteurs, y compris les lecteurs de la traduction. Ainsi, la traduction constitue aussi une «pré compréhension », une «pré-structure» de la compréhension encore plus profonde (si nous excluons les éléments socio-économiques, c'est justement la raison de la retraduction). La traduction doit être aussi un «texte scriptible» ouvert à ses lecteurs; elle doit attendre, avec une attitude amicale, la prochaine compréhension (la retraduction, comme nous l'avons déjà dit).

Le développement de la linguistique moderne nous dévoile encore l'arbitraire de la langue et nous indique que cet arbitraire ne se limite pas seulement au lien unissant le signifiant au signifié. II peut nous amener jusqu'à l'intraduisible qui reste toujours un des grands problèmes de la traduction. (Ici, nous n'avons pas besoin de répéter les points de vue de Humboldt qui pense même que la pensée humaine est décidée par la langue.) En ce cas, la seule chose qui puisse nier l'intraduisible, c'est, comme «l'histoire effective» proposée par Gadamer, l'efficacité relative de la langue. Cette efficacité est aussi historique. À moins que nous ne la mettions dans le cours de I'histoire, la langue ne deviendra jamais efficace. Le texte original, lui-même, porte aussi cette empreinte historique, il survit en subissant tous les changements historiques. Le préjugé est juste, c'est-à-dire que l'existence du traducteur dans la traduction est non seulement inévitable, mais aussi raisonnable. Cequi devrait être mis en cause, c'est au contraire la fidélité absolue que Milan Kundera préconise. Ce que nous traduisons, ce n'est pas, non plus, un objet qui est en opposition à «nous» - ce sujet spirituel - , mais une relation, une concordance entre l'auteur et le traducteur. II est déjà reconnu de tous que si la science de la linguistique ne peut pas inclure la théorie de la traduction, c'est justement parce qu'elle ne peut pas expliquer de façon claire 
cette vieille dichotomie objet / sujet qui empêche aussi le développement de la linguistique elle-même. Elle ne veut pas reconnaître qu'un texte original peut produire différents effets à différentes époques, et, surtout au niveau de la traduction, selon différents traducteurs de différentes époques. Ces différences participent à la création de l'histoire. Donc toute compréhension, notamment l'acte du traduire, n'est jamais une copie du texte original. Si nous pouvons accepter la proposition de Gadamer, la traduction est aussi la fusion des deux horizons. Tout en reconnaissant qu'il existe une distance indissimulable entre l'horizon de l'auteur et celui du traducteur, nous pouvons dire que la tâche de la traduction ne consiste pas à les faire disparaître, mais tout au contraire, à les faire fusionner, c'est-à-dire à créer un nouvel horizon qui comprend le «précipité» de l'histoire. Ce processus de fusion n'est pas la simple addition, et le nouvel horizon existe en tant que «tout», qui deviendrait le point de départ de la prochaine compréhension.

Personne ne peut nier les valeurs de la traduction. Pourtant les valeurs de la traduction ne résident pas dans la fidélité: on a besoin de la traduction seulement parce qu'elle ne manque pas d'esprit de création et qu'elle peut faire partie de la littérature de la langue d'arrivée. Ainsi, tout en signalant la nécessité de la traduction, la notion de recréation voudrait aussi justifier la traduisibilité en précisant ce qu'on recrée dans la traduction littéraire. Si l'on ne peut pas préciser ce à quoi on doit être fidèle, on peut essayer, de toute façon, de déterminer ce qu'on recrée dans la traduction.

La question se pose tout d'abord au niveau du lexique. La recréation veut dire travailler l'impossible. À ce niveau, cette recréation se présente principalement sous forme d'emprunt ou de réorganisation des mots et expressions existant déjà dans la langue d'arrivée. Nous disons toujours que l'opération traduisante est un processus de compromis. C'est-à-dire qu'en général nous choisissons, dans la langue d'arrivée, des mots ayant des champs sémantiques proches de ceux de la langue de départ. Cela implique que la transformation de sens est presque toujours inévitable: nous conservons même les mots et expressions de la langue d'arrivée, mais nous leur donnons déjà de nouvelles significations. C'est l'arbitraire de la relation entre signifiant et signifié qui décide. Les mots restent les mêmes, mais la relation fixée entre signifiant et signifié du texte original est brisée.

Bien sûr, le cas le plus typique est celui de l'emprunt. Henri M eschonnic définit même toute la traduction comme «un grand emprunt». En effet, c'est une définition du côté fonctionnel de la traduction. La différence entre les champs sémantiques des deux systèmes de langue provoque naturellement certaines lacunes conceptuelles dans la langue d'arrivée. Et à ce moment-là, la traduction les emplit souvent à l'aide de l'emprunt. Edward Said propose ainsi la notion de «voyage théorique», et l'opération traduisante, tout à fait comme la définition d'Henri M eschonnic, devient tout d'abord le transport de ce fameux voyage. Et ce voyage a justement pour commencement - si nous pouvons emprunter, nous aussi, le langage de Said - «le voyage lexique».

Cette notion de «voyage lexique» n'en est pas moins intéressante pour notre théorie de la traduction. Le problème que Said nous laisse, c'est que le lexique de la langue de départ (et le concept ou l'idéologie aussi) ne voyage jamais lui-même, et la traduction n'est absolument pas un simple moyen de transport de ce voyage. La traduction est un endroit où se produit un champ sémantique du lexique tout neuf, 
différent de celui de la langue de départ, mais aussi différent de celui existant déjà dans la langue d'arrivée. C'est le traducteur qui réalise cette pratique translinguistique et transculturelle.

Citons l'exemple de la traduction au début du $x x^{e}$ siècle. C'est à ce moment que la civilisation traditionnelle de la Chine a été complètement bouleversée par celle de I'Europe occidentale. En ce qui concerne la langue, cela se présente tout d'abord sous la forme de l'utilisation d'un nouveau lexique. Étant une langue pictographique tout à fait différente des langues occidentales, le chinois n'a pas pu emprunter directement leur lexique. Donc il existe toujours un problème de nationalisation du lexique étranger. Aujourd'hui, par exemple, peut-être qu'on ne doute plus de la relation réciproque entre «self» et «自我》, car le dictionnaire le stipule ainsi. M ais cette relation n'a été fixée qu'au début de ce siècle, au cours du deuxième essor de la traduction en Chine. Et, si nous remontons à l'origine de ce mot dans le confucianisme, nous ne serons pas moins étonnés de voir la différence entre les deux champs conceptuels de «自我 》 à différentes époques. Ainsi, comme nous l'avons déjà indiqué, c'est la traduction qui crée, pour elle-même, un espace de langage ayant sa propre histoire. C'est aussi la traduction qui donne à l'ancien lexique une occasion d'être expliqué autrement et qui, en plus, atteste cette nouvelle signification.

Ensuite, le problème de la recréation se pose aussi au niveau syntaxique. Dans les grammaires traditionnelles, la syntaxe est souvent subordonnée à la morphologie. M ais les linguistes modernes y voient, en quelque sorte, I'épine dorsale de la langue. Si les grammaires traditionnelles se tournent enfin vers la syntaxe, c'est parce qu'elles ne peuvent pas expliquer clairement la relation d'interaction entre les unités lexicales de la phrase. Et c'est justement en s'appuyant sur cette relation d'interaction que la syntaxe peut intervenir de manière décisive dans la signification d'une phrase: «Le chat mange la souris» et «La souris mange le chat» n'ont certainement pas le même sens, la dernière phrase peut exister grammaticalement, mais pas syntaxiquement. Parce que la relation syntaxique implique aussi une relation logique.

Dans notre traduction, la syntaxe contribue tout d'abord à l'interprétation relativement exacte du texte, puis à la recréation mesurée du texte original dans un autre système de langage, car la syntaxe constitue l'efficacité provisoire et le côté statique de la langue où réside notre traduisible. Le traducteur doit, certainement, acquérir les connaissances de la syntaxe de la langue de départ et aussi de celle de la langue d'arrivée.

Et ce n'est pas tout. Ce qui est le plus paradoxal et a le lien le plus étroit avec la notion de recréation, c'est que malgré sa rigueur, la syntaxe a aussi, et c'est même une de ses caractéristiques importantes, une certaine ambiguïté. Intentionnellement ou non, il ne manque pas de cette sorte d'ambiguïté dans un texte littéraire et ces sortes d'ambiguïtés se présentent à l'étape de la compréhension aussi bien qu'à l'étape de la ré-expression. Ces différentes interprétations provoquent naturellement différentes traductions. M ais une part considérable du sens échappe à la syntaxe, en particulier le sens lexical garde le même sens, et nous n'hésitons pas, à ce moment-là, à demander secours à la sémantique qui s'appuie à la fois sur une sémantique lexicale, indépendante de la syntaxe, et sur une sémantique non lexicale, celle qui résulte de l'association des unités lexicales dans une même phrase. En effet, la sémantique nous fait voir le côté exact de la langue; toutefois, si nous croyons que nous pouvons tout résoudre à l'aide de la sémantique, nous risquons d'être trompés. Parce que l'auteur d'un texte 
littéraire est toujours prêt à transgresser les règles grammaticales et ainsi à utiliser «justement» ces ambiguïtés de la syntaxe. Le cas le plus représentatif est certainement la poésie dont nous parlons beaucoup dans le littéraire. Conserver ces ambiguïtés intentionnelles de l'auteur n'apparaît que comme un rêve si nous considérons la syntaxe comme une simple forme du contenu et que nous ne la traitons pas au niveau de la recréation. Toute prétention de les éclaircir peut avoir un résultat catastrophique.

En réalité, l'intraduisible n'existe que lorsque la fonction référentielle de la langue a tout à fait disparu, ou presque, et ainsi la syntaxe, comme partie indispensable de la sémantique, perd elle aussi sa fonction.

Nous devons reconnaître que, dans certains cas, les poèmes sont presque intraduisibles. Mais c'est justement parce que la syntaxe n'a aucun sens dans les poèmes et que la recréation dont nous parlons ne peut se fonder que sur la base linguistique. L'efficacité provisoire de la langue est gravement compromise dans ce cas. Et, dans la recréation de la traduction littéraire, la syntaxe, malgré son indépendance, n'est qu'une partie d'un tout. Elle doit se soumettre, avec le lexique, au besoin de «littérarité» du texte littéraire.

En plus du lexique et de la syntaxe, la prosodie reste aussi un des éléments importants de la littérature. Son importance est plutôt due à son lien direct avec le niveau esthétique du texte littéraire. Ainsi, la musicalité de Flaubert ou la longue phrase de Proust constitue souvent la marque spéciale de l'écrivain et entre dans son système de style.

Le structuralisme a essayé d'intégrer la prosodie au système structural du texte littéraire. En effet, bien qu'elle fasse partie du style, que nous considérons depuis longtemps comme intraduisible, elle peut être analysée comme un élément mesurable du texte.

Le rythme, la pause et l'intonation sont les trois éléments principaux de la prosodie dont l'existence implique, étymologiquement, ce qui, dans le langage, se rapproche du chant. Si nous remontons à l'origine de la littérature, nous serons étonnés de découvrir que toute «littérature» est restée longtemps essentiellement orale. Les textes ont été tout d'abord récités ou chantés par les aèdes, les jongleurs et les ménestrels. Cela prouve l'importance de la poésie dans presque toutes les grandes civilisations. C'est en ce sens que nous devons considérer peut-être le niveau esthétique comme le niveau le plus original de la littérature. II consiste à éveiller les cinq sens de I'homme et ainsi à accomplir sa fonction poétique.

Le problème de la prosodie nous semble important, car il nous fait remarquer que - si nous reconnaissons que la musique est en quelque sorte sans frontières la littérature est aussi sans frontières. L'homme est toujours sensible à tout ce qui peut susciter son imagination, c'est la base fondamentale de notre traduction. Mais, comme nous l'avons déà indiqué, la sensibilité de l'homme est aussi variable selon son expérience, sa situation culturelle et même son niveau d'éducation. Et, en réalité, la prosodie «d'un texte écrit doit toujours être reconstituée, à partir des pauses des groupes syntaxiques, des intentions expressives prêtées à l'écrivain ou propres à l'interprète» (M illy 1992: 192).

Si nous faisons une comparaison entre les éléments prosodiques de deux langues, même de différentes familles de langues, nous trouverons toujours quelques ressemblances. Presque toutes les littératures connaissent ces trois éléments: rythme, pause et intonation. Donc ce n'est absolument pas la «forme» de la poésie qui est 
intraduisible. Et la reconstitution de la prosodie du texte original à l'aide des moyens prosodiques de la langue d'arrivée est toujours possible.

Bien sûr, nous n'avons pas la prétention de discuter spécialement de traduction poétique dans cet article. En plus de la versification, il existe d'autres problèmes comme l'image et le thème - dans la traduction poétique. II nous semble nécessaire aussi d'indiquer que la prosodie n'est pas non plus une spécialité de la poésie. Elle est commune à tous les genres littéraires et même à des textes non littéraires. Nous voudrions seulement démontrer que, en tant que genre original de la littérature, et même fixée de manière rigoureuse, la poésie, classique ou moderne, possède aussi une prosodie transmissible. Nous croyons que la recréation n'est qu'au niveau esthé tique, qui est le niveau le plus élevé de la littérature. M ais ce n'est pas tout à fait vrai. Le niveau esthétique est le niveau le plus fondamental de tous les arts, notamment de la littérature. C'est aussi en ce sens-là que nous pouvons dire que la traduction littéraire est une recréation, non seulement selon sa base philosophique, mais aussi du côté de la poétique.

Enfin, il nous reste encore un problème un peu plus délicat: la recréation du style. En effet, il est difficile de concevoir un texte dépourvu de tout style, même s'il s'agit d'un texte non littéraire. Le problème du style nous semble vraiment important, surtout dans la traduction littéraire. Car le système de style est directement lié aux éléments esthétiques du texte.

Notre première question concernant le style est celle-ci : le style de l'auteur est-il recréable?

La réponse pourrait être simple: si le style peut être analysé, il peut être recréé. La plupart des théoriciens de la traduction adoptent l'attitude qui consiste à séparer le sens du style, et ainsi de poser d'abord le sens, puis le style. Cependant, si nous considérons le style comme sacrifiable, le contenu ne peut non plus être préservé. Comment pouvons-nous dire que les éléments qui entrent dans le système de style, comme le lexique, la syntaxe et la prosodie, nefont pas partie du contenu proprement dit? Donc ce n'est pas à partir de tout cela que nous pouvons recréer le style de l'auteur et, à vrai dire, ce n'est pas la recréation que nous proposons. Nous disons que le style de l'auteur est recréable, et aussi au niveau esthétique. Que ce soit l'intention personnelle de l'auteur ou la marque empreinte sur «la langue interne», elles sont textes unifiés au niveau esthétique et deviennent ainsi inséparables.

Pourtant, ce qui embarrasse vraiment le traducteur, ce n'est peut-être pas la possibilité de recréer le style de l'auteur mais, tout au contraire, l'existence inévitable du style du traducteur lui-même.

Nous avons déjà démontré que la littérature n'est pas la simple déférence aux prescriptions communes des pratiquants d'une langue, elle est composée de ce qui la distingue des autres formes d'écriture, sa «littérarité». Cela implique que tout texte littéraire, notamment la traduction d'un texte littéraire, comporte certainement l'empreinte personnelle de son créateur.

En reconnaissant la créativité du traducteur, nous reconnaissons bien l'existence du style du traducteur.

II est sans doute plus clair dans la retraduction. Si la traduction doit être «transparente» par rapport au texte original et si l'interprétation absolument objective existe, alors la retraduction n'existera plus. $M$ ais malheureusement, le phénomène de retraduction existe toujours et devient même de plus en plus fréquent. 
Certains traducteurs se demandent (bien sûr, et nous demandent) : Si l'auteur écrivait son œuvre en langue d'arrivée, comment écrirait-il?

C'est une question amusante, mais sans réponse, car l'auteur n'écrira jamais son œuvre en langue d'arrivée. Sinon la traduction n'existerait pas.

II est vrai que les différents traducteurs pourraient interpréter, par différents côtés et de différentes façons, le style de l'auteur. C'est quand même compréhensible. Le style, en tant que système supérieur du texte, consiste aussi, comme les autres systèmes du texte, en l'arbitraire de la relation entre signifiant et signifié. Une fois le texte accompli, cette relation est fixée d'une certaine manière et, dans la littérature, d'après l'intention de l'auteur. Le traducteur prend contact avec le texte tout d'abord comme un lecteur, il brise cette relation et l'interprète selon sa compréhension. Quand il commence à le traduire, il s'efforce de reconstituer une nouvelle relation entre signifiant et signifié dans la langue d'arrivée. Ce qui distingue le traducteur de l'écrivain, c'est qu'il joue deux fois sur l'arbitraire de la relation entre signifiant et signifié, et surtout dans deux systèmes de langage.

Donc nous devons savoir que le style du traducteur est inévitable. Un texte sans style n'existe pas; et un texte sans style personnel de l'auteur n'est pas un texte littéraire.

Enfin, il nous reste une seule question qui est pourtant la plus importante: quelle est la relation entre le style de l'auteur du texte original et celui du traducteur? La réponse à cette question concerne aussi directement le degré de la recréation dont nous allons parler spécifiquement. Bien que le traducteur soit toujours discret devant son propre style, nous lisons toujours la trace du traducteur, «ce qui n'est pas de l'auteur».

Guo Hongan, un des traducteurs chinois du Rouge et le Noir explique ainsi ce qu'il pense du problème de cette relation: le traducteur doit choisir un écrivain proche de lui-même. Tout en préservant sa propre personnalité, il ne trahit quand même pas l'auteur du texte original, c'est pourquoi dans presque tous les pays, il existe toujours des traducteurs qui traduisent spécialement tel ou tel écrivain.

\section{LA RECRÉATION DANS LA FIDÉLITÉ : DEGRÉS DE RECRÉATION}

Ainsi nous considérons la notion de recréation comme une conception complémentaire de la notion de fidélité. La fidélité est une condition préalable de la recréation, et la recréation peut se concrétiser, dans la pratique traduisante, par des recréations de degrés différents. Nous croyons que le traducteur peut adopter trois attitudes vis-àvis du texte original : supériorité, égalité et infériorité. La plupart des traducteurs acceptent la dernière au nom de la fidélité. Comme Meschonnic (1982) I'indique dans son ouvrage: «La plupart des traducteurs ne sont pas des "créateurs". La traduction vieillit - pourquoi ? Là où le texte qu'elle traduit ne vieillit pas - pourquoi ? et on le retraduit - pourquoi ?» Pourquoi ? Parce que nous sommes enchaînés par cette notion absolue de fidélité.

Malheureusement, l'égal ité absolue n'existe pas, elle n'existe que théoriquement. Les exemples de certains traducteurs en tant que «créateurs» ne témoignent que du succès de la supériorité au texte original. Nous avons, en France, par exemple, 
Diderot, Mallarmé, Nerval et Baudelaire; en Angleterre, Pound; et en Chine, Lin Shu - «Les meilleurs traducteurs ont été écrivains» (M eschonnic 1982).

C'est vrai. II semble que toutes les grandes œuvres aient été celles qui portent les marques personnelles les plus évidentes. En tant que partie indispensable de la litté rature, la traduction littéraire ne fait pas exception.

Si nous avons déjà fait une analyse générale sur la recréation des grands éléments d'un texte littéraire, c'est-à-dire du côté de la langue et du style, nous devons nous demander encore s'il existe vraiment, comme Barthes (1970) l'indique, «une autre réalité» qui se trouve entre la langue et le style. Car :

langue et style sont des données antécédentes à toute problématique du langage; langue et style sont le produit naturel du Temps et de la personne biologique; mais l'identité formelle de l'écrivain ne s'établit véritablement qu'en dehors de l'installation des normes de la grammaire et des constantes du style, là où le continu écrit, rassemblé et enfermé d'abord dans une nature linguistique parfaitement innocente, va devenir enfin un signe total, le choix d'un comportement humain, l'affirmation d'un certain bien, engageant ainsi l'écrivain dans l'évidence et la communication d'un bonheur ou d'un malaise, et liant la forme à la fois normale et singulière de sa parole à la vaste Histoire d'autrui.

Nous devons peut-être poser la question d'une autre façon quand nous discutons du degré de la recréation dans la traduction littéraire. Nous ne voulons pas diviser la traduction, comme les autres théoriciens le font, en «mot à mot», «littérale» et «libérale». Nous ne voulons pas, non plus, tout en préconisant l'une d'entre elles, condamner les autres. Ce que nous voulons faire c'est, plutôt comme Roland Barthes, chercher «une autre réalité» qui peut diviser, plus objectivement, la traduction en certains degrés. Et si la langue est en deçà de la littérature, et le style presque au-delà, notre degré de recréation peut aller du niveau de la langue jusqu'au niveau du style, et même au-dessous du niveau de la langue et au-dessus du niveau du style.

Avec les trois attitudes du traducteur, il existe certainement trois degrés de recréation : recréation au degré bas, recréation au degré moyen et recréation au degré élevé. Et, en plus, nous considérons l'intraduisible comme le degré zéro de la recréation. Pour une œuvre donnée dans un rapport interlinguistique-interculturel donné, l'intraduisible peut exister. Mais c'est seulement parce que «l'interaction des poétiques et la réénonciation historique» ne se sont pas encore produites. Ce qui décide des degrés de la traduction, c'est la fonction de la traduction et la demande du lecteur de la traduction.

En général, nous croyons que la traduction a pour but final la communication éventuelle entre différentes nations qui pratiquent différentes langues. C'est vrai, nous traduisons, malgré toutes les difficultés que nous rencontrons, parce que nous avons envie de connaître les autres, et que nous avons envie d'être connus par les autres.

Cependant, quelles sont les fonctions concrètes que la traduction assume? Certes, elle est le moyen principal de l'échange culturel. Nous parlons ici de l'échange, nous pouvons simplement le considérer comme une action de «transporter dans notre littérature une œuvre étrangère (ancienne ou non); nous transporter dans l'autre littérature (dans son passé, ou non) » (M eschonnic 1982). C'est là que réside la plupart des traductions qui se contentent de raconter une histoire exotique. Ce point de vue peut amener la traduction dans deux directions contraires. D'une part, le 
traducteur pense qu'il doit être fidèle au texte original, au contenu tout d'abord et, si c'est possible, à la forme ensuite. Dans ce cas-là, le contenu (I'intrigue et la description) devient naturellement la plus importante chose que le traducteur doit s'efforcer de représenter. Et,

si par coïncidence il est possible de véhiculer le même contenu dans la langue d'arrivée dans une forme qui ressemble étroitement à celle de départ, tant mieux; nous conservons la forme quand nous pouvons, mais le plus souvent la forme doit être transformée précisément pour conserver le contenu. (Nida 1964)

D'autre part, c'est-à-dire quand le traducteur est obligé de sacrifier la fidélité à la forme pour conserver le contenu, il va souvent plus loin, jusqu'à la réécriture. Ces deux cas sont des cas extrêmes de la recréation : la recréation au degré le plus bas et la recréation sans degré.

Mais Henri Meschonnic comprend la fonction de la traduction d'une manière tout à fait différente: sa fonction «est d'être une transformation poétique et culturelle». Cette transformation est, au sens de Heidegger (dans M eschonnic 1982) :

par la traduction, le travail de la pensée se trouve transposé dans l'esprit d'une autre langue et subit ainsi une transformation inévitable. Mais cette transformation peut devenir féconde, car elle fait apparaitre en une lumière nouvelle la position fondamentale de la question.

En fait, c'est dans la traduction que les deux cultures se heurtent, se complètent. M ais si nous acceptons le « décentrement » d'H enri M eschonnic, la traduction fournit aussi des ressources à la langue d'arrivée. C'est à ce niveau-là que la fonction de la traduction ne consiste plus dans l'information, mais dans la valeur.

Cela peut sans doute expliquer pourquoi «les meilleurs traducteurs ont été des écrivains». Prenons encore comme exemple le $x x^{e}$ siècle en Chine: il est vrai qu'au début de ce siècle, la plupart des traducteurs chinois étaient des écrivains. Ils ont apporté, à la langue d'arrivée, non seulement de nouveaux genres littéraires, mais aussi une nouvelle structure linguistique. Nous devons parler encore, dans cette partie, de la notion d'historicité de la traduction. La traduction est toujours historique; si les meilleurs traducteurs ont été des écrivains, c'est parce qu'ils sont apparus à l'époque où une langue allait voir sa fin et avait besoin de se renouveler de fond en comble. Cela fournit souvent à la traduction une bonne occasion de s'affirmer. Quand une langue ne se satisfait plus, elle demande à la traduction plus de créativité qui pourrait la stimuler. Ainsi Jackson Mathews (1966: 68) pense que «chaque langue a ses propres formes, actuelles et possibles: celles que les poètes ont déjà trouvées et utilisées, et celles qui sont encore à trouver». Donc la traduction, considérée comme un moyen révélant les possibilités cachées d'une langue, met l'accent plutôt sur la créativité. Nous réalisons, de cette manière, l'aventure non seulement d'un sujet historique, mais aussi d'une structure linguistique.

Ainsi, certains savants ont même indiqué, ces dernières années, que dans la diffusion de l'eurocentrisme et la formation du langage postcolonialiste, c'est la traduction qui joue le rôle le plus important. Car c'est la traduction qui intègre le lexique (et l'idéologie aussi) à sa propre langue; si, au nom de la fidélité, le traducteur reste à la position du texte original, le nouveau langage qui se forme à partir de la traduction porte inévitablement cette empreinte. 
Et le lecteur joue aussi un rôle important dans les différents degrés de la traduction. Si nous reconnaissons que le littéraire est une partie de la littérature, et même une manière spéciale de création littéraire proprement dite, nous ne pouvons pas négliger l'existence du lecteur qui décide d'une certaine manière de l'attitude et du choix du traducteur, et ainsi du degré de la recréation. Le lecteur fait partie de I'histoire, et s'il fait partie de l'histoire de la littérature, il fait certainement partie de I'histoire de la traduction littéraire.

En effet, «l'écriture et la lecture (en traduction, c'est la traduction et la lecture de la traduction) sont en relation dialogique». Peut-être que notre traducteur était trop attentif à l'auteur du texte original, qu'il a oublié totalement qu'il existait encore un lecteur derrière lui ! Comme nous l'avons déjà indiqué, la traduction n'est qu'une station dans la circulation de la compréhension. La traduction littéraire, outre qu'elle procure un plaisir particulier, entraîne aussi d'autres réactions textuelles, dans la retraduction, chez les critiques et les autres traducteurs. C'est aussi une des raisons principales de la retraduction, c'est-à-dire qu'elle peut aussi susciter une insatisfaction chez les autres traducteurs. Et, en plus, les fonctions de la traduction littéraire, quelque complexes qu'elles soient, se fondent aussi sur cette lecture active.

Face au fait qu'il existe inévitablement une transformation au cours du processus «pensée - signifiant de la langue de départ - signifié (représentation mentale) signifiant de la langue d'arrivée - signifié (représentation mentale que le lecteur de la traduction crée) », si nous ne voulons pas perdre confiance, il vaut mieux partir du dernier maillon de ceprocessus: le lecteur. Nous pourrions bien lui demander ce qu'il attend de la traduction. Lui aussi, il est un des points de départ de notre critique du littéraire. Et si nous voulons estimer la traduction à sa juste valeur, nous ne pouvons pas ignorer tout cela.

La réponse à cette question peut être contraire à ce que nous nous figurons. $C^{\prime}$ 'est justement le cas de la discussion sur la traduction chinoise du Rouge et le Noir dont les lecteurs semblent préférer la recréation au degré moyen (soi-disant convenable). La notion de «concurrence entre les deux langues et les deux cultures» de Xu Yuanchong a provoqué une grande contestation non seulement chez les autres traducteurs, mais aussi chez les critiques et les lecteurs qui se sentent trahis par le traducteur. Les lecteurs attendent toujours de la traduction quelque chose d'exotique qui peut se distinguer de leur propre langue, de leur propre culture.

Si nous pouvons comprendre que la culture est un système ouvert, nous comprendrons l'attente de nos lecteurs. Tout cela, avec la fonction que la traduction va remplir, définit les limites et les raisons d'être des degrés de la recréation. C'est justement la raison pour laquelle nous appelons notre recréation la recréation dans la fidéité. Ainsi pouvons-nous appeler aussi notre fidélitéla fidélité dans la recréation. La belle infidèle de M. M énage n'aurait aucun sens, puisque nous ne croyons pas que la traduction n'a qu'un seul maître - le texte d'origine. 


\section{NOTE}

1. Voir Xu Jun: «Réflexions sur les études des problèmes fondamentaux de la traduction», dans le présent numéro.

\section{RÉFÉRENCES}

Al bir , Amparo Hurtado (1990) : La Notion de fidélité en traduction, Paris, Didier Érudition.

Barthes, Roland (1970) : S/Z, Paris, Seuil.

Gen et t e, Gérard (1966) : «Structuralisme et critique», Figures I, Paris, Seuil.

Lad mir al , Jean-René (1994) : Traduire: théorèmes pour la traduction, Paris, Gallimard.

M an, Paul de (1986) : The Resistance to Theory, M inneapolis, University of M inneapolis Press.

M at h ew s, Jackson (1966) : «Third Thought on Translating Poetry», dans On Translation, Oxford,

Oxford University Press.

Meschonnic, Henri (1982) : Pour la poétique II, Paris, Gallimard.

M il ly, Jean (1992) : Poétiques des textes, Paris, Nathan.

M o un in, Georges (1955) : Les Belles Infidèles, Paris, Éditions des Cahiers du Sud.

Nida, Eugène (1964) : Toward a Science of Translation, Leyde, Brill.

Saussu r e, Ferdinand de (1968) : Cours de linguistique générale, texte établi par Bally et Sechehaye,

Paris, Payot.

Xu, Jun (1992) : Études sur la critique de la traduction littéraire, Nanjing, Éditions Yilin. 